\title{
Continuous Renal Replacement Therapy among Patients with COVID-19 and Acute Kidney Injury
}

\author{
Ryann Sohaney ${ }^{\mathrm{a}}$ Salma Shaikhouni ${ }^{\mathrm{a}}$ John Travis Ludwig ${ }^{\mathrm{a}}$ Anca Tilea $^{\mathrm{b}}$ \\ Markus Bitzer $^{\mathrm{a}}$ Lenar Yessayan ${ }^{\mathrm{a}}$ Michael Heung ${ }^{\mathrm{a}}$ \\ aDivision of Nephrology, Department of Internal Medicine, University of Michigan, Ann Arbor, MI, USA; \\ bepartment of Obstetrics and Gynecology, University of Michigan, Ann Arbor, MI, USA
}

\section{Keywords}

Acute kidney injury · Continuous renal replacement therapy · Coronavirus disease 2019 . Acute respiratory distress syndrome

\begin{abstract}
Background and Objectives: Acute kidney injury (AKI) is a common complication among patients with COVID-19 and acute respiratory distress syndrome. Reports suggest that COVID-19 confers a pro-thrombotic state, which presents challenges in maintaining hemofilter patency and delivering continuous renal replacement therapy (CRRT). We present our initial experience with CRRT in critically ill patients with COVID-19, emphasizing circuit patency and the association between fluid balance during CRRT and respiratory parameters. Design, Setting, Participants, and Measurements: Retrospective chart review of 32 consecutive patients with COVID-19 and AKI managed with continuous venovenous hemodiafiltration with regional citrate anticoagulation (CVVHDF-RCA) according to the University of Michigan protocol. Primary outcome was mean CRRT circuit life per patient during the first 7 days of CRRT. We used simple linear regression to assess the relationship between patient characteristics and filter life. We also explored the relationship between fluid balance on CRRT and respiratory parameters
\end{abstract}

karger@karger.com

(C) 2021 S. Karger AG, Basel

www.karger.com/bpu

Karger! using repeated measures modeling. Results: Patients' mean age was 54.8 years and majority were Black (75\%). Comorbidities included hypertension $(90.6 \%)$, obesity $(70.9 \%)$ diabetes $(56.2 \%)$, and chronic kidney disease (40.6\%). Median CRRT circuit life was 53.5 [interquartile range 39.1-77.6] hours. There was no association between circuit life and inflammatory or pro-thrombotic laboratory values (ferritin $p=$ 0.92 , C-reactive protein $p=0.29$, D-dimer $p=0.24)$, or with systemic anticoagulation $(p=0.37)$. Net daily fluid removal during the first 7 days of CRRT was not associated with daily (closest recorded values to $20: 00) \mathrm{PaO}_{2} / \mathrm{FIO}_{2}$ ratio $(p=0.21$ ) or positive end-expiratory pressure requirements $(p=0.47)$. Conclusions: We achieved adequate CRRT circuit life in COVID-19 patients using an established CVVHDF-RCA protocol. During the first 7 days of CRRT therapy, cumulative fluid balance was not associated with improvements in respiratory parameters, even after accounting for baseline fluid balance.

(c) 2021 S. Karger AG, Basel

\section{Introduction}

Early reports of disease related to the novel COVID-19 from China described a relatively low incidence of acute kidney injury (AKI): $0.5 \%$ in all cases and $2.9 \%$ in severe cases [1]. However, subsequent reports from the United 
States have suggested higher incidence of AKI in hospitalized patients of $28-37 \%[2,3]$. Among patients with COVID-19 and AKI, a significant proportion required acute dialysis, ranging from 20 to over 50\% [3-5]. As a result, COVID-19 related surges in patients with AKI have resulted in potential shortages in renal replacement therapy (RRT) availability $[5,6]$.

Continuous RRT (CRRT) in patients with COVID-19 poses special challenges. Epidemiological studies have described patients with significant coagulopathy [7], and it has been suggested that anticoagulation may improve outcomes [8]. Patients with COVID-19 and dialysis requiring AKI (AKI-D) have higher peak D-dimer and inflammatory markers than those without or with less severe AKI [9] which further implicate a pro-thrombotic state. The increased risk of thrombosis in patients with COVID-19 may affect CRRT hemofilter life span. In turn, premature hemofilter clotting diminishes the efficiency of dialysis, consumes potentially scarce resources, and increases nursing staff workload and exposure to COVID-19. The 2012 Kidney Disease Improving Global Outcomes (KDIGO) AKI guideline recommends regional citrate anticoagulation (RCA) in patients who are not already receiving systemic anticoagulation to minimize circuit clotting [10]. However, standards for CRRT anticoagulation vary widely across institutions, and there has been little report on CRRT anticoagulation strategy in patients with COVID-19.

Fluid management in COVID-19 affected patients with acute respiratory distress syndrome (ARDS) can also be a challenge. Fluid overload may contribute to worsening respiratory status and achieving a net negative fluid balance with CRRT could theoretically help improve hypoxia. Studies of patients with ARDS suggest that a conservative fluid management strategy leads to reduced time on mechanical ventilation and in the intensive care unit (ICU). However, patients with AKI were excluded from these trials and fluid balance was adjusted using diuretics and not dialysis. Conversely, aggressive volume removal may jeopardize hemodynamics and lead to adverse consequences. As such, there remains significant uncertainty regarding the optimal approach to volume management in patients with AKI-D and ARDS.

We report our initial experiences with COVID-19 patients who developed ARDS and AKI requiring CRRT. The aims of this study were to quantify CRRT hemofilter life and to additionally identify factors associated with premature hemofilter clotting. We further sought to describe the effect of fluid balance during CRRT on respiratory parameters.

CRRT among Patients with COVID-19

Infection

\section{Materials and Methods}

Study Population, Setting, and Data Collection

We performed a retrospective chart review of the first 32 consecutive adult patients (18 years or older) with COVID-19 and AKI-D who underwent CRRT, corresponding to the period from March 17, 2020, to April 12, 2020, at the University of Michigan. Only patients with a confirmed COVID-19 infection as the cause for admission to the ICU were included for analysis. A confirmed COVID-19 infection was defined as a positive viral nucleic acid reverse transcriptase-polymerase chain reaction assay collected on a nasopharyngeal swab or tracheal aspirate. We excluded patients with end-stage renal disease on maintenance dialysis prior to hospital admission, although patients with a functioning kidney allograft were included.

During the study period, CRRT was performed exclusively with the Prismaflex CRRT machine (Baxter Inc., Deerfield, IL, USA) in continuous venovenous hemodiafiltration (CVVHDF) mode with RCA using anticoagulant citrate dextrose solution A. CVVHDF-RCA was prescribed according to an institutional protocol: prescribed effluent dose of approximately $25 \mathrm{~mL} / \mathrm{kg} / \mathrm{h}$, fixed blood flow $(100-150 \mathrm{~mL} / \mathrm{min})$ to anticoagulant citrate dextrose solution A (250-300 mL/h) rate, and target post-filter circuit ionized calcium of $0.2-0.4 \mathrm{mmol} / \mathrm{L}$. RCA was prescribed unaltered for all CVVHDF circuits, irrespective of systemic anticoagulation exposure. Calcium-free dialysate and replacement solutions were used, and replacement fluid was delivered post filter. Calcium chloride infusion was adjusted to maintain systemic ionized calcium in the 1.05-1.25 $\mathrm{mmol} / \mathrm{L}$ range. Access for RRT was placed at the bedside by the primary critical care team.

Demographic data, comorbid conditions, and clinical and laboratory data were manually abstracted by review of the electronic medical record. Data collection was censored at death or hospital discharge.

\section{Study Variables and Definitions}

Comorbid conditions were ascertained by physician documentation. Chronic kidney disease was additionally ascertained by eGFR $<60 \mathrm{~mL} / \mathrm{min} / 1.73 \mathrm{~m}^{2}$ or albuminuria in the year prior to hospital admission. Onset of symptoms was obtained by manual abstraction from the admission History and Physical documentation.

RRT start was defined as CRRT initiation or intermittent hemodialysis (IHD) initiation in patients undergoing IHD prior to CRRT. The laboratory data most proximal to CRRT initiation (and within $48 \mathrm{~h}$ ) was selected to reflect acuity of illness at the time of CRRT initiation. For patients receiving IHD prior to CRRT, the basic metabolic panel variables were recorded as most proximal to IHD initiation as these are often altered directly by the IHD procedure. The partial pressure of oxygen $\left(\mathrm{PaO}_{2}\right)$ to fraction of inspired oxygen $\left(\mathrm{FiO}_{2}\right)$ ratio $(\mathrm{P} / \mathrm{F})$ and positive end-expiratory pressure (PEEP) most proximal to CRRT initiation and their daily values (closest value to 20:00) for the first 7 days of CRRT were recorded. This time was chosen to avoid selecting a $\mathrm{P} / \mathrm{F}$ value that may have occurred during a spontaneous breathing trial which is typically done in the morning.

CRRT circuit life, the time between CRRT circuit initiation and circuit interruption, and reason for interruption were obtained from nursing documentation in a CRRT flow sheet for each CRRT circuit initiated in the first 7 days of CRRT. Possible reasons for interruption included "hemofilter clotting," "hemofilter expired," "machine error," "procedure or imaging," or "CRRT discontin- 
ued." Hemofilter life was defined separately as the time to circuit interruption inclusive of hemofilter clotting and expiration only.

The Institutional Review Board at the University of Michigan approved this study with a waiver for informed consent due to the retrospective observational design (HUM00029545). The study used existing data obtained during routine health care and standard documentation, with no additional testing, procedures, or patient contact.

\section{Statistical Analysis}

Descriptive statistics were used to summarize the data. Numeric variables were reported as mean and standard deviation and median and interquartile range (IQR) as appropriate. Categorical variables were summarized as frequency and proportion. We determined the circuit life of all CRRT circuits started within the first 7 days of CRRT. To report circuit life at the patient level, we first calculated the mean circuit life for each individual patient. We additionally report circuit life by individual circuit. We assessed for an association between systemic anticoagulation use and dialysis access site (right internal jugular [RIJ] vs. other) with circuit life with Wilcoxon rank-sum. We used simple linear regression to assess the relationship between baseline inflammatory markers (ferritin, D-dimer, C-reactive protein (CRP), and circuit life.

To assess the relationship between fluid balance on CRRT and oxygenation, we used repeated measures modeling with daily cumulative fluid balance on CRRT as an independent variable and $\mathrm{P} / \mathrm{F}$ as the dependent variable, and adjusted for fluid accumulation at CRRT start, height, weight, and age. The same methodology was used to assess the association between cumulative fluid balance on CRRT and PEEP. Analyses were performed with SAS 9.4 (SAS Institute Inc., Cary, NC, USA).

\section{Results}

\section{Patient Population}

From March 17, 2020, to April 12, 2020, 32 of 194 (16.5\%) patients with confirmed COVID-19 infection admitted to the ICU at the University of Michigan experienced AKI-D and were initiated on CVVHDF-RCA. Patient demographics, comorbid conditions, and hospitalization characteristics appear in Table 1 . The mean age was 54.8 years and $75 \%$ of patients were Black. Patients had a high burden of comorbid disease with $90.6 \%$ of patients having hypertension, $70.9 \%$ obesity, $56.2 \%$ diabetes mellitus, $40.6 \%$ chronic kidney disease, and $18.8 \%$ prior solid organ transplantation. Twenty-three (71.9\%) patients received systemic anticoagulation with unfractionated heparin. All CVVHDF circuits were anticoagulated with RCA irrespective of systemic anticoagulation exposure. All patients required mechanical ventilation.

The majority (57.1\%) of patients met criteria for AKI on admission. The median time from symptom onset to RRT initiation was 8.0 days [IQR 6.0-12.8] and the median time from hospital admission to RRT initiation was
Table 1. Characteristics of 32 patients with COVID-19 infection and AKI requiring CRRT

\begin{tabular}{|c|c|}
\hline \multicolumn{2}{|l|}{ Demographics } \\
\hline Age, years & $54.8 \pm 10.7$ \\
\hline \multicolumn{2}{|l|}{ Race } \\
\hline Black & $24(75)$ \\
\hline White & $6(18.8)$ \\
\hline Other/unknown & $2(6.3)$ \\
\hline \multicolumn{2}{|l|}{ Comorbid conditions } \\
\hline Diabetes mellitus & $18(56.2)$ \\
\hline Hypertension & $29(90.6)$ \\
\hline Obesity $(\mathrm{BMI}>30.0)$ & $23(71.8)$ \\
\hline CKD & $13(40.6)$ \\
\hline Cardiovascular disease & $9(28.1)$ \\
\hline Asthma or COPD & $11(34.4)$ \\
\hline Liver cirrhosis & $1(3.1)$ \\
\hline Solid organ transplant & $6(18.8)$ \\
\hline Baseline SCr $(n=26), \mathrm{mg} / \mathrm{dL}$ & $1.5 \pm 0.77$ \\
\hline Baseline eGFR $(n=26), \mathrm{mL} / \mathrm{min} / 1.73 \mathrm{~m}^{2}$ & $67 \pm 26$ \\
\hline \multicolumn{2}{|l|}{ Hospitalization characteristics } \\
\hline \multicolumn{2}{|l|}{ Systemic anticoagulation } \\
\hline Unfractionated heparin & $23(71.9)$ \\
\hline None & $9(28.1)$ \\
\hline Mechanical ventilation & $32(100)$ \\
\hline Extracorporeal membrane oxygenation & $2(6.3)$ \\
\hline Admission $\mathrm{SCr}, \mathrm{mg} / \mathrm{mL}$ & $2.3[1.4-4.1]$ \\
\hline Onset of symptoms to RRT initiation $(n=30)$, day & $8.0[6.0-12.8]$ \\
\hline Admission to RRT start, day & $4.0[1.5-7.0]$ \\
\hline \multicolumn{2}{|l|}{ Initial RRT modality } \\
\hline IHD & $7(21.9)$ \\
\hline CRRT & $25(78.1)$ \\
\hline Oliguria $^{a}$ & $28(87.5)$ \\
\hline \multicolumn{2}{|l|}{ CV SOFA } \\
\hline $0-2$ & $10(31.3)$ \\
\hline $3-4$ & $22(68.8)$ \\
\hline Cumulative fluid balance at CRRT initiation, $\mathrm{L}$ & $3.3[2.0-5.6]$ \\
\hline $\mathrm{P} / \mathrm{F}$ at $\mathrm{CRRT}$ initiation & $137[94-172]$ \\
\hline \multicolumn{2}{|l|}{ CRRT access type } \\
\hline RIJ & $19(59.4)$ \\
\hline Other ${ }^{c}$ & $13(40.6)$ \\
\hline
\end{tabular}

Values for continuous variables are expressed as mean \pm SD or median [IQR]. Values for categorical variables are expressed as count (\%). SD, standard deviation; BMI, body mass index; COPD, chronic obstructive pulmonary disease; $\mathrm{SCr}$, serum creatinine; eGFR, estimated glomerular filtration rate; IQR, interquartile range; $\mathrm{RRT}$, renal replacement therapy; CRRT, continuous renal replacement therapy; CV SOFA, cardiovascular sequential organ assessment score; $\mathrm{P} / \mathrm{F}$, ratio of arterial oxygen partial pressure $\left(\mathrm{PaO}_{2}\right)$ to fraction of inspired oxygen $\left(\mathrm{FiO}_{2}\right)$; $\mathrm{CKD}$, chronic kidney disease; RIJ, right internal jugular; $\mathrm{AKI}$, acute kidney injury; IHD, intermittent hemodialysis. ${ }^{a}$ Oliguria is urine output $<0.5 \mathrm{mg} / \mathrm{kg} / \mathrm{h}$ for at least $6 \mathrm{~h}$ prior to the onset of RRT. ${ }^{\mathrm{b}} \mathrm{CV}$ SOFA, 0, MAP $\geq 70 \mathrm{~mm} \mathrm{Hg} ; 1$, MAP $<70 \mathrm{~mm} \mathrm{Hg} ; 2$, dopamine $\leq 5 \mu \mathrm{g} /$ $\mathrm{kg} / \mathrm{min}$ or dobutamine; 3 , dopamine $>5 \mu \mathrm{g} / \mathrm{kg} / \mathrm{min}$ or epinephrine $\leq 0.1 \mu \mathrm{g} / \mathrm{kg} / \mathrm{min}$ or norepinephrine $\leq 0.1 \mu \mathrm{g} / \mathrm{kg} / \mathrm{min} ; 4$, dopamine $>15$ $\mu \mathrm{g} / \mathrm{kg} / \mathrm{min}$ or epinephrine $>0.1 \mu \mathrm{g} / \mathrm{kg} / \mathrm{min}$ or norepinephrine $>0.1$ $\mu \mathrm{g} / \mathrm{kg} / \mathrm{min}$. ${ }^{c}$ Other access incudes left internal jugular vein, femoral vein, and extracorporeal membrane oxygenation circuit. 
Table 2. Laboratory data at RRT start

\begin{tabular}{llll}
\hline & $N$ & Laboratory value & Reference range \\
\hline $\mathrm{SCr}, \mathrm{mg} / \mathrm{dL}$ & 32 & $5.3 \pm 2.1$ & $0.70-1.30$ \\
Sodium, mmol/L & 32 & $138[134-142]$ & $136-146$ \\
Potassium, mmol/L & 32 & $4.9 \pm 0.6$ & $3.5-5.0$ \\
Blood urea nitrogen, $\mathrm{mg} / \mathrm{dL}$ & 32 & $67[48-94]$ & $8-20$ \\
$\mathrm{Phosphorous}, \mathrm{mg} / \mathrm{dL}$ & 32 & $6.7 \pm 2.5$ & $2.7-4.6$ \\
$\mathrm{pH}$ & 32 & $7.28 \pm 0.08$ & $7.35-7.45$ \\
$\mathrm{PaO}, \mathrm{mm} \mathrm{Hg}$ & 32 & $84.0 \pm 32.0$ & $80-100$ \\
$\mathrm{PCO}, \mathrm{mm} \mathrm{Hg}$ & 32 & $43.3 \pm 9.7$ & $35-45$ \\
$\mathrm{HCO}, \mathrm{mmol} / \mathrm{L}$ & 32 & $19.0[17-23]$ & $22-26$ \\
$\mathrm{Lactate}, \mathrm{mmol} / \mathrm{L}$ & 32 & $1.7 \pm 1.0$ & $0.5-2.2$ \\
Total bilirubin, $\mathrm{mg} / \mathrm{dL}$ & 32 & $0.6[0.4-1.25]$ & $0.2-1.2$ \\
$\mathrm{AST}, \mathrm{IU} / \mathrm{L}$ & 32 & $63[44-130]$ & $8-30$ \\
$\mathrm{ALT}, \mathrm{IU} / \mathrm{L}$ & 32 & $38[25-105]$ & $\leq 35$ \\
Albumin, $\mathrm{g} / \mathrm{dL}$ & 32 & $3.1 \pm 0.5$ & $3.5-4.9$ \\
Ferritin, $\mathrm{ng} / \mathrm{mL}$ & 32 & $1,587[933-2,219]$ & $18.0-32.0$ \\
$\mathrm{CRP}, \mathrm{mg} / \mathrm{dL}$ & 32 & $20.0[8.6-31.3]$ & $0-0.6$ \\
Procalcitonin, $\mathrm{ng} / \mathrm{mL}$ & 31 & $4.3[0.9-6.1]$ & $0-0.25$ \\
$\mathrm{hs}$ troponin, $\mathrm{pg} / \mathrm{mL}$ & 20 & $47[33-95]$ & $0-19$ \\
$\mathrm{D}-\mathrm{dimer}, \mathrm{mg} / \mathrm{L}$ & 31 & $6.0[3.0-9.5]$ & $<0.59$ \\
$\mathrm{LDH}, \mathrm{IU} / \mathrm{L}$ & 23 & $592[489-910]$ & $120-240$ \\
\hline
\end{tabular}

Values are presented as mean $\pm \mathrm{SD}$ or median [IQR]. SCr, serum creatinine; RRT, renal replacement therapy; AST, aspartate aminotransferase; ALT, alanine transaminase; LDH, lactate dehydrogenase; IU, international unit; CRP, C-reactive protein; SD, standard deviation; $\mathrm{IQR}$, interquartile range.

4.0 days [IQR 1.75-7.0]. The most common RRT access site was RIJ vein $(n=19)$, followed by femoral vein $(n=$ $8)$, left internal jugular vein $(n=4)$, and extracorporeal membrane oxygenation circuit $(n=1)$.

Laboratory data prior to RRT start are presented in Table 2. Elevation in inflammatory markers at CRRT initiation was common. Median ferritin was 1,583 [IQR 933-2,219] $\mathrm{ng} / \mathrm{mL}$ and median CRP was 20.0 [IQR 8.633.0] $\mathrm{mg} / \mathrm{dL}$. Elevation in $\mathrm{D}$-dimer was also common with a median D-dimer of 6.0 [IQR 2.0-9.5] mg/L.

\section{CRRT Circuit and Hemofilter Life}

A total of 164 patient days and 74 CRRT circuits were analyzed across 32 patients. The median CVVHDF circuit life per patient was 53.5 [IQR 39.1-77.6] hours (Fig. 1). When excluding hemofilters that were taken down for reasons other than clotting or routine expiration, the median hemofilter life per patient was 65.3 [IQR 15.9-94.0] hours. When examining all filters individually, the median circuit life was 51.8 [IQR 15.9-92.7] hours. Sixteen (21.6\%) filters clotted within $24 \mathrm{~h}$ of initiation, of which 11 were recorded as being related to vascular access issues. Conversely, $47 \%$ of filters achieved $60 \mathrm{~h}$ or greater life span.

CRRT among Patients with COVID-19 Infection
Median circuit life was 44.3 [IQR 37.8-68.4] hours for those treated with systemic anticoagulation $(n=23)$ and 75.3 [IQR 49.8-82.5] hours for those without $(n=9)$, which was not a statistically significant difference $(p=$ $0.37)$. Median $\mathrm{D}$-dimer was higher among patients receiving systemic anticoagulation $(7.1 \mathrm{mg} / \mathrm{L}, \mathrm{IQR} 4.3-$ 10.4) than those without $(2.4 \mathrm{mg} / \mathrm{L}$, IQR $1.3-5.6)(p=$ 0.02 ). When comparing circuit life by access site, there was no difference between patients with a RIJ access versus any other access location (median 53.8 vs. $53.2 \mathrm{~h}, p=$ 0.86 ). In simple linear regression models, there was no significant association between circuit life and any inflammatory or coagulopathy makers (ferritin $p=0.92$, CRP $p=0.29$, D-dimer $p=0.24$ ).

\section{CRRT Fluid Balance and Respiratory Parameters}

The median cumulative fluid balance from admission to CRRT start was +3.3 [IQR 2.0-5.6] L. There was a trend toward increasingly negative fluid balance on CRRT (Fig. 2a) whereas there was variation, but no clear trend in daily $\mathrm{P} / \mathrm{F}$ (Fig. 2b) and PEEP (Fig. 2c). When adjusting for age, weight, height, and cumulative fluid balance at CRRT initiation, there was no association between daily cumulative fluid balance on CRRT and P/F $(p=0.21)$ or PEEP $(p=0.47)$. 
Fig. 1. Mean hemofilter life per patient during the first 7 days of continuous renal replacement therapy.

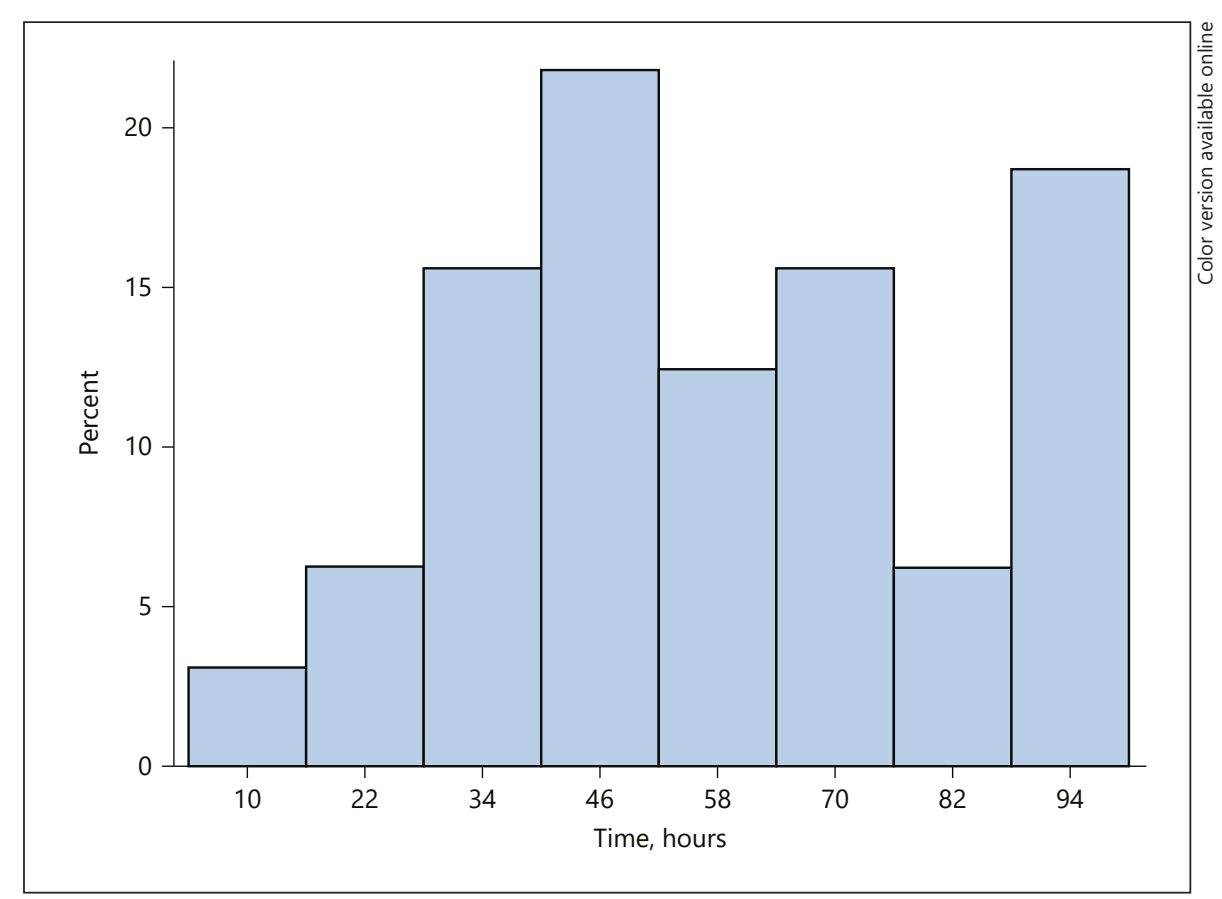

\section{Patient Outcomes}

The median ICU length of stay $(n=32)$ was 19.5 (IQR 12.5-29.0) days and median hospital length of stay $(n=$ 32) 32.0 (IQR 13.0-47.5) days. Nineteen (59.4\%) patients survived to hospital discharge; of which 13 recovered sufficient kidney function to be liberated from RRT prior to hospital discharge. Among survivors, the median length of mechanical ventilation $(n=19)$ was 20.0 (IQR 12.033.0) days.

\section{Discussion}

The COVID-19 pandemic surge in 2020 resulted in large number of patients requiring acute dialysis. To date, there has been little report of the AKI-D experience in these patients, although anecdotal reports have noted clotting issues with CRRT, which have the potential to exacerbate already limited supplies of dialysis disposables. In this study, we describe our initial experience providing CRRT to 32 critically ill patients with COVID-19 and AKI. Importantly, we found that CVVHDF with RCT was effective in maintaining CRRT circuit patency. In addition, we observed that fluid removal using CRRT during the first 7 days of CRRT did not correlate with an improvement in respiratory parameters.

During the initial surge in COVID-19 infections, the University of Michigan experienced a rapid increase in nephrology consultations for AKI among critically ill patients. Similar to experiences elsewhere $[6,11]$ this led to a dramatic surge in utilization of CRRT, which became a potentially scarce resource requiring careful monitoring of both machine and disposable usage rates. Anecdotally, nephrologists in several institutions have reported increased hemofilter clotting and shorter circuit patency among critically ill patients with COVID-19 [12], which are additional threats to availability of CRRT disposables. Our study, however, demonstrates that our RCT protocol was effective in maintaining circuit patency comparable to our non-COVID-19 experience. Although we did not have a noncitrate anticoagulation comparison group, our observed median circuit survival of $53.5 \mathrm{~h}$ is superior to what has been reported in recent randomized controlled trials (RCTs) comparing anticoagulation strategies in CRRT. Schilder and colleagues compared RCA to heparin in patients on CRRT and reported median filter survival times of 46 and $32 \mathrm{~h}$, respectively [13]. In a similar trial, Stucker and colleagues noted mean CRRT filter life spans of $49 \mathrm{~h}$ with RCA and $28 \mathrm{~h}$ with heparin [14]. In the largest RCT, including 596 patients, Zarbock and colleagues also demonstrated significantly longer median filter survival using an RCA strategy than systemic heparin (47 h vs. $27 \mathrm{~h}$ ) [15]. In our experience, having an effective anticoagulation protocol to avoid premature circuit clotting was pivotal to meeting the demand for CRRT and minimizing disposable waste. 
Fig. 2. Box plots of daily trends in fluid balance from CRRT start (a), PEEP (b), and partial pressure of oxygen $\left(\mathrm{PaO}_{2}\right)$ to fraction of inspired oxygen $\left(\mathrm{FiO}_{2}\right)$ ratio $(\mathbf{c})$. The bottom and top of the boxes span the 25th (Q1) and 75th (Q3) percentile. Medians are represented by horizontal lines and mean by squares in the middle of the boxes. The whiskers indicate 1.5 times the IQR from Q1 and Q3. Data points falling outside this range are additionally displayed. CRRT, continuous renal replacement therapy; PEEP, positive end-expiratory pressure; IQR, interquartile range.

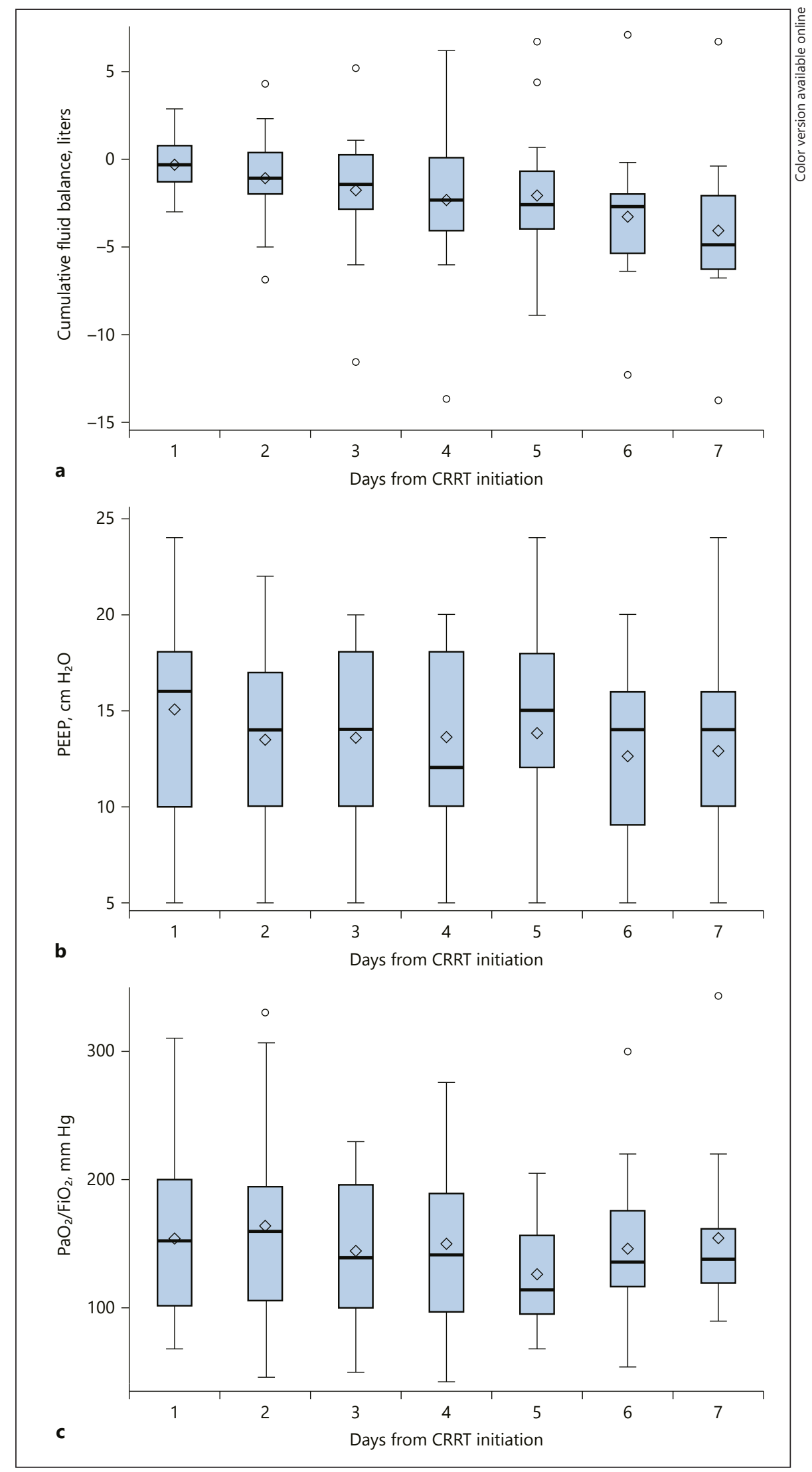

Blood Purif 2022;51:660-667 
There are several potential explanations for the extended CRRT circuit life observed in our study. During the time of this study, patients with COVID-19 suspected of having deep venous thrombosis were treated empirically with systemic anticoagulation, and confirmatory doppler venous ultrasound and CT scans were deferred to reduce staff and equipment exposures. As a result, most patients in this cohort received systemic heparin in addition to RCA for CRRT. However, patients receiving RCA alone actually exhibited a trend toward higher circuit survival times than patients receiving both heparin and RCA (although not statistically significant), indicating that the favorable circuit survival was not driven by the high proportion of systemic heparin use within our cohort. Therefore, in absence of an indication for systemic anticoagulation, we do not recommend dual anticoagulation as a routine strategy for prolonging CRRT circuit life. Another important factor is that our institution has been utilizing RCA for CRRT since 2002 with a high volume of CRRT patients [16]. This accumulated expertise among both physicians and nursing staff facilitates troubleshooting of circuit issues and efforts to optimize filter life span. While we support broader use of RCA for CRRT as recommended by KDIGO guidelines [10], we also recognize that centers with little or no experience using RCA will necessarily require training to establish and implement an RCA protocol, and such resources may not be available during times of stress such as the COVID-19 pandemic.

As reported in many other studies, patients with COVID-19 were observed to have significant elevations in inflammatory and coagulopathy markers. However, we did not find an association between ferritin, CRP, or Ddimer and circuit survival. As such, use of such markers may not be an effective approach to identifying patients who are at highest risk for clotting, and therefore not helpful to determining anticoagulation approach. Although we did not have a nonanticoagulated group, based on the anecdotal reports of premature clotting we support recent expert recommendations to pursue some form of anticoagulation for CRRT over no anticoagulation [12].

A major advantage to the use of CRRT in critically ill patients with AKI is the ability to manage volume status through continuous ultrafiltration. While there are no RCT of optimal fluid management strategies with CRRT, a restrictive approach to fluid management has been advocated in ARDS. The Fluid and Catheter Treatment Trial (FACTT) of 1,000 patients with ARDS reported that a restrictive fluid strategy was associated with more ventilator-free days [17]. However, patients requiring RRT were excluded from the trial and fluid balance was managed with diuretics. In the present study, we observed a gradual trend toward negative fluid balance over the first 7 days of CRRT secondary to net ultrafiltration. However, change in volume balance was not associated with any significant improvement in respiratory parameters, even after adjusting for fluid balance at CRRT initiation. It is possible that the effect of ultrafiltration on respiratory status in ARDS is not immediately apparent and may require longer periods of observation to become apparent. Similarly, the modest amount of fluid removal observed during this study may have been inadequate to result in clinically apparent improvements. Conversely, the observed positive fluid balance may have been an inaccurate assessment of fluid overload in this patient population, particularly since some patients with COVID-19 will have gastrointestinal symptoms and may present in a volume-depleted state. Clearly additional fluid removal in such patients would not be beneficial and could in fact be harmful. As such, careful assessment of volume status remains an important aspect of the management of patients with COVID-19, and an individualized approach should be taken to determining ultrafiltration strategy in this population.

In our initial experience with patients with COVID-19 and AKI requiring CRRT, we were able to achieve circuit life spans comparable to our experience with non-COVID-19 patients and longer than generally reported in the literature. While we were not able to compare to other anticoagulation strategies, citrate appears to be an effective anticoagulation strategy in this patient population.

\section{Statement of Ethics}

The Institutional Review Board at the University of Michigan approved this study with a waiver for informed consent due to the retrospective observational design (HUM00029545). The study was performed ethically in accordance with the World Medical Association Declaration of Helsinki.

\section{Conflict of Interest Statement}

The authors have no conflicts of interest to declare.

\section{Funding Sources}

Drs. Sohaney and Ludwig were funded by training grants from the National Institutes of Health 5T32DK007378-40 and 1T32AG062403-01A1, respectively. Additional support was provided by the Michigan Institute for Clinical and Health Research (Clinical and Translational Science Award, CTSA: UL1TR002240). 


\section{Author Contributions}

Study concept and design were contributed by R.S., S.S., J.T.L., M.H., and L.Y.; data acquisitions were contributed by R.S., S.S., and J.T.L.; data analysis and/or interpretation were contributed by all authors. Critical revision of the manuscript for important intellectual content was contributed by all authors.

\section{Data Availability Statement}

The data that support the findings of this study are not publicly available due to their containing information that could compromise the privacy of research participants.

\section{References}

1 Guan WJ, Ni ZY, Hu Y, Liang WH, Ou CQ, $\mathrm{He}$ JX, et al. Clinical characteristics of coronavirus disease 2019 in China. N Engl J Med. 2020;382(18):1708-20.

2 Hirsch JS, Ng JH, Ross DW, Sharma P, Shah $\mathrm{HH}$, Barnett RL, et al. Acute kidney injury in patients hospitalized with COVID-19. Kidney Int. 2020;98(1):209-18.

3 Mohamed MMB, Lukitsch I, Torres-Ortiz AE, Walker JB, Varghese V, HErnandez-Arroyo $\mathrm{CF}$, et al. Acute kidney injury associated with coronavirus disease 2019 in urban New Orleans. Kidney360. 2020;1(7):614-22.

4 Gupta S, Hayek SS, Wang W, Chan L, Mathews KS, Melamed ML, et al. Factors associated with death in critically Ill patients with coronavirus disease 2019 in the US. JAMA Intern Med. 2020;180(11):1436-47.

5 Fisher M, Prudhvi K, Brogan M, Golestaneh L. Providing care to patients with acute kidney injury and COVID-19 infection: experience of front line nephrologists in New York. Kidney360.. 2020;1(6):544-8.

6 Division of Nephrology, Columbia University Vagelos College of Physicians. Disaster response to the COVID-19 pandemic for patients with kidney disease in New York City. J Am Soc Nephrol. 2020;31(7):1371-9.
7 Zhang Y, Xiao M, Zhang S, Xia P, Cao W, Jiang $\mathrm{W}$, et al. Coagulopathy and antiphospholipid antibodies in patients with covid-19. N Engl J Med. 2020;382(17):e38.

8 Paranjpe I, Fuster V, Lala A, Russak A, Glicksberg BS, Levin MA, et al. Association of treatment dose anticoagulation with in-hospital survival among hospitalized patients with $\mathrm{CO}$ VID-19. J Am Coll Cardiol. 2020;76(1):122-4.

9 Mohamed MMB, Lukitsch I, Torres-Ortiz AE, Walker JB, Varghese V, HErnandez-Arroyo $\mathrm{CF}$, et al. Acute kidney injury associated with coronavirus disease 2019 in urban New Orleans. Kidney360. 2020;1(5).

10 Pandharipande PP, Girard TD, Jackson JC, Morandi A, Thompson JL, Pun BT, et al. Long-term cognitive impairment after critical illness. N Engl J Med. 2013;369(14):1306-16.

11 Goldfarb DS, Benstein JA, Zhdanova O, Hammer E, Block CA, Caplin NJ, et al. Impending shortages of kidney replacement therapy for COVID-19 patients. Clin J Am Soc Nephrol. 2020;15(6):880-2.

12 Nephrology Journal Club blog (Neph JC). Acute Kidney Injury. Available from: http://www. nephjc.com/news/covidaki. Accessed $2020 \mathrm{Jul}$ 25.

13 Schilder L, Nurmohamed SA, Bosch FH, Purmer IM, den Boer SS, Kleppe CG, et al. Citrate anticoagulation versus systemic heparinisation in continuous venovenous hemofiltration in critically ill patients with acute kidney injury: a multi-center randomized clinical trial. Crit Care. 2014;18(4):472.
14 Stucker F, Ponte B, Tataw J, Martin PY, Wozniak H, Pugin J, et al. Efficacy and safety of citrate-based anticoagulation compared to heparin in patients with acute kidney injury requiring continuous renal replacement therapy: a randomized controlled trial. Crit Care. 2015;19:91.

15 Zarbock A, Küllmar M, Kindgen-Milles D, Wempe C, Gerss J, Brandenburger T, et al. Effect of regional citrate anticoagulation vs systemic heparin anticoagulation during continuous kidney replacement therapy on dialysis filter life span and mortality among critically Ill patients with acute kidney injury: a randomized clinical trial. JAMA. 2020;324(16): 1629-39.

16 Swartz R, Pasko D, O'Toole J, Starmann B. Improving the delivery of continuous renal replacement therapy using regional citrate anticoagulation. Clin Nephrol. 2004;61(2): $134-43$.

17 National Heart, Lung, and Blood Institute Acute Respiratory Distress Syndrome (ARDS) Clinical Trials Network; Wiedemann HP, Wheeler AP, Bernard GR, Thompson BT, Hayden D, et al. Comparison of two fluidmanagement strategies in acute lung injury. N Engl J Med. 2006;354(24):2564-75. 